\title{
Study on the Positioning of Sports Humanities Sociology
}

\author{
Hui Zhang \\ School of Physical Education, Xinjiang University, Urumqi Xinjiang, 830008, China
}

Keywords: Sports humanities and social studies, Main content, Positioning

\begin{abstract}
In the sports humanities and social sciences, the most important thing is to combine human phenomena and social phenomena, compared with other disciplines, sports humanities and social sciences more scientific, with strong application, the author positioning Study of Sports Humanities and Social Sciences is to be able to better reveal the fundamental nature of sport in social phenomena, its own characteristics, system configuration, and other laws of the formation, which is more important is sports humanities and sociology of science with various relationships, including: sports science, humanities, science education, national sciences in interdisciplinary research in the humanities and social sports, all kinds of positioning for the development of national sports teaching has a recessive impact, we should face up to the meaning of positioning to lay the foundation for the development of the country.
\end{abstract}

\section{Introduction}

Humanities and social sciences in sports development process, or there are more deficiencies related academics but also its effective research, to shorten the gap with other countries, recognizing that sports in the humanities and social sciences basis points, according to the theory Research based on the relationship between various scientific disciplines and sports, and to make its proper positioning, on this basis, to ensure the positioning of Sports Humanities and social sciences more scientific and reasonable, according to the relevant definitions and content of sports humanities and social sciences research to ensure that the construction of sports humanities and social sciences can be more reasonable.

\section{Research of sports social phenomena}

In sports humanities and social sciences research, sports phenomenon is more important research basis, social phenomena is beyond everyone's consciousness, it is a method of inhibiting the urging of his actions, in the process, which has the objective, the existence of a strong, high research value of this social phenomenon, the main point of view is divided into the following areas:

First, in the humanities and social science research in sports, sports phenomenon is part of society, is a dominant presence in the individual, and the individual's body instinctively different reactions, such as: people's psychological reaction, genetic characteristics, hobbies, etc. This outside Existence is not sufficient to form a social phenomenon, not the achievements of the social phenomenon of sports, research shows that the existence of this phenomenon has a more beneficial effect on the development of sports disciplines [1].

Second, the social phenomenon is the product of a collective sport features in sports phenomenon in which a person's performance does not constitute a social phenomenon, all activities must be collective, it can be called sports social phenomenon. For example: in sports, there is a group of people wearing clothes the color is rather special, this is not a sports social phenomenon, only everyone wear the same clothes, it belongs to the sports social phenomenon. The emergence of this social phenomenon, have an impact on the development of sports disciplines, could make it more scientific, standardized, toward a better direction [2].

Thirdly, in the sports social science research, social phenomena are more compelling, mainly because sport phenomenon determined by the majority of people, most people concentrated form of will and faith, accept the edification of others unknowingly, and with a power to guide people to accept, to develop the same habits. For example: people set the rules, people form collective habits, 
and others will comply with the relevant provisions of the unconscious in the basketball game process. Another example: in the social riots in non-sports phenomenon, people do not want to participate in a collective atmosphere would be affected, participate in them, these are the characteristics of social phenomena. Social phenomenon generated by the impact of the collective activities, but also by the collective activity hard to instill in others, making it a member of the collective.

Fourth, in the sports social phenomena, Sports and Physical discipline is directly related to the social phenomenon of sport is more special, is more extensive, is an application of the strong influence of broader social phenomenon, greater impact the development of sports disciplines.

Fifth, in the performance of the process of social phenomena in sports, social phenomenon is a special form in front of people, along with a wide range of sports disciplines in the development of education, so that countries gradually national road, which is a favorable development of the country influence, sport is no longer just used to improve people's quality of their own, exercise the way, has begun to become a national sport in the key elements [3].

\section{Property of sports humanities and social studies}

\section{Strong integrity}

In interdisciplinary research in the humanities and social sports, sports humanities and social sciences are more integrated discipline, from multiple angles, aspects; various features reflect sports humanities and social phenomenon, specifically apparent from the following aspects:

First, in the humanities and social science research sports in the process, need to be the object of study all angles, all aspects of the investigation, to be able to relax their vision, meticulous nuanced assessment study, does not have any flaws. On this basis, but also to the overall situation, we can not look at a single object of study, to it as a sport as a whole, from the study who analyzed the existing problems in order to achieve a reasonable analysis, sum up experience, to take effective measures for the purpose of better able to grasp the situation [4].

Second, in sports humanities and social science research, it can be found in the knowledge that it contains more are potential in sports humanities and social science knowledge, such as: knowledge of literature, history knowledge, legal knowledge, artistic knowledge, knowledge management The presence of these knowledge sufficient to prove a comprehensive knowledge of the humanities and social sciences sport, more valuable and significance in research.

Thirdly, in sports humanities and social science research process, research staff will perform the work in various ways, not only according to each type of things, each branch point, characteristics of each content, and take effective measures for its research studies, but also to Research using a more integrated approach major and difficult issues, in a dynamic way to deal with dynamic problems with static methods for static problems, both macro and micro, can meet the appropriate study conditions [5].

\section{Strong theoretical property}

Interdisciplinary research in humanities and social sport, which has a more obvious feature is the theory of strong, mainly for the following aspects: first, during the study of sports humanities and social phenomena, should pay attention to its own nature, sports, humanities and social the phenomenon itself is derived from the humanities and social performance of the two phenomena, awareness of their formation is more profound, one can go from perception to rational summary of relevant experience, law society is completely reflected. Second, sports humanities and social science research and philosophy there is a certain correlation, humanities and social sciences in sports development process, honed and growth for many years, has become strong enough theoretical discipline, scholars should be relevant for sports humanities and social development of discipline and make the appropriate summary, to avoid difficult problems arise gradually update. 


\section{Strong application}

In sports humanities and social sciences research, sports science discipline is strong, compared with the traditional sports humanities, it has become a social branches in various disciplines in the development process, it is vulnerable to challenge the community Some scholars of sports culture is not enough understanding of social phenomena, which blindly divided in narrow disciplines, resulting in a more adverse impact on their development. In fact, in today's rapid development of science and technology, sports, humanities and social sciences knowledge has become more popular, so the relevant researchers should make more effective the views according to their own research.

\section{Strong scientific nature}

In sports humanities and social sciences research, sports science discipline is strong, compared with the traditional sports humanities, it has become a social branches in various disciplines in the development process, it is vulnerable to challenge the community Some scholars of sports culture is not enough understanding of social phenomena, which blindly divided in narrow disciplines, resulting in a more adverse impact on their development. In fact, in today's rapid development of science and technology, sports, humanities and social sciences knowledge has become more popular, so the relevant researchers should make more effective the views according to their own research.

\section{Strong humanity}

Sports humanities and social sciences in the study, there is a strong humanities, mainly because such a culture is summed up summed up based on human observation and research, according to people in sports activities, the performance of lessons learned, which class natural sciences and physical characteristics are different signs. Direct it, humanities is based on the human spirit and life observations to human biology as a benchmark for sports humanities and social science research, so that it can get better development.

\section{Positioning of sports humanities and social studies}

\section{Relation between sports science}

By comparison between sports humanities and social sciences and physical education can be found, belonging to sports humanities and social sciences branch sports disciplines, we can clearly show the development of sports science, but still has some limitations. Sports humanities and social sciences and physical education are cross-cutting and penetration of relationship, on the basis of the relationship between the penetrations, some knowledge is the same, such as: the principle of sport, the original body, teaching and research direction. However, the two also has a place of mutual restraint, sports humanities and social sciences influenced the development of sports science, not better enhance the level of development, and sports humanities and social sciences have failed to mature, cannot be separate from physical education development, which causing a contradiction between the two, so that researchers can better study it.

\section{Relation between humanistic and social science}

In sports humanities and social sciences research process, we can see sports humanities and social sciences are more complex, which has a complementary relationship between the sport and the humanities and social sciences, humanities and social sciences in sport, a lot of theory and by the humanities Social science theory is introduced, and its purpose is to solve practical problems occurring in sports, then there this bifurcation theory sports humanities and social sciences. Meanwhile, in the humanities and social sciences, humanities and social sciences sport belongs to the humanities and social sciences, "child", not even "weaned", sports humanities and social sciences has not yet developed into a more mature, stable discipline, it is still dependent on the survival of Humanities and Social Sciences it is the study of the main issues that need attention. In addition, in the process of sports humanities and social science research, the humanities and social sciences as its parent show, should pay attention to research and application of humanities and social science theory, 
with its unique concept of sports science to solve problems, broad application of theoretical knowledge in order to achieve its purpose rich experience and results.

\section{Relation between other second-class disciplines}

In sports humanities and social sciences, there are many affiliated secondary subjects, including: Physical education, training, science, traditional science, the emergence of these two subjects, is conducive to research staff from different angles of sports humanities and social sciences research, we can use the theory of two disciplines, etc., expand the appropriate sports science research. In this process, stakeholders should be aware that the humanities and social sciences humanities and social sciences are the sports integral system, these two subjects in macro perspective, theory and concepts are cross-cutting, they can learn from each other, offset from each other, and even to some extent, the common use of some theories and concepts, which sports disciplines humanities and social studies are more favorable. In addition, researchers also according to the characteristics related to other two disciplines for effective adjustments to sports humanities and social sciences, and will meet development needs.

\section{Relation between sports social science}

During the study in the humanities and social sports disciplines, the natural sciences can be divided into the following major areas: the natural, social and human sciences three categories, these three areas are the main points related research division, in its mutual comparison process, differences in social, cultural sciences is very small, while others are relatively large difference, for now sports humanities and social sciences, the social sciences sport is more important, in the course of the study, presented more often Effective reference relationship, which the positioning of sports humanities and social sciences are more favorable, complementary relationship between the various disciplines, and sufficient to improve the efficiency of humanities and social studies sports disciplines, so that it can better applications in sports problem is solved. In addition, in three disciplines, the research staff of sports humanities and social sciences should be targeted based on their theories and concepts, body positioning and the development of sports science at this time has a greater need for contact, in the humanities and social sports disciplines positioning, not only to increase the research staff of sports social science research efforts, but also need it in different situations, drawing knowledge from different disciplines, to ensure better positioning sports humanities and social sciences, their application in physical education laid basis.

\section{Conclusions}

With the development of the country under, sports humanities and social sciences for the development of sports undertakings exerting teaching role in promoting sports-related social science is the basis of the theory of sports reform, for sports humanities and social sciences, in the case of research-oriented positioning, to make greater efforts to improve its international effect, increasing development in the world today, sports humanities and social sciences can easily become the world's main scientific research, personnel must examine its position based on the relationship between science and the humanities and social sciences other sports .

\section{Acknowledgement}

This paper is the National Social Science Fund Youth Project, Project Name: Study on countermeasures for Xinjiang schools carrying out sunshine sports for the heritage and development of minority traditional sports, the approval number: 09CTY011; also it is a school level teaching reform project, Project Name: Phase 4 project of Xinjiang University "21st Century Higher education teaching reform project" - Xinjiang University Gym sports course teaching practice and reform study", Number: XJU2015JGY79. 


\section{References}

[1] Huang Juyun, Li Yin, Cha Anni. Comparative analysis of Sports humanistic sociology and sociology master's thesis - and innovation of study model of China 's Sports Humanities and Sociology.Journal of Shanghai University Of Sport,2013,37(6):7-13.

[2] Qu Jie, Wang Yi. Topics direction and methods characteristic of China's sports humanistic sociology studies - analysis of doctoral thesis majored in Sports Humanistic Sociology between 1999 - 2011. Journal of Shanghai University Of Sport,2013,37(5):13-17.

[3] Zhu Hui, Deng Sanhong, Wang Hao. Sports Humanistic Sociology disciplines introduce network building and analysis. Library and Information, 2013(6):77-83.

[4] Han Jiebing. Sports Humanistic Sociology paper cooperation study and analysis. Journal of Southwest University for Nationalities(humanities \& social sciences edition),2013(12):213-224.

[5] Li Rui. Thesis writing issues of Sports Humanistic Sociology paper - centered in published in journals.China Sports Science, 2014(6):122-128. 